\title{
EVALUATION OF THE PERFORMANCE OF THE HYBRID LATTICE BOLTZMANN BASED NUMERICAL FLUX
}

\author{
H. W. ZHENG \\ LHD, Institute of Mechanics, Chinese Academy of Science \\ No.15 Beisihuanxi Road, Beijing 100190, China \\ h.zheng@imech.ac.cn \\ C. SHU \\ National University of Singapore, 10 Kent Ridge Crescent, Singapore 119260 \\ mpeshuc@nus.edu.sg
}

Published 22 June 2016

\begin{abstract}
It is well known that the numerical scheme is a key factor to the stability and accuracy of a Navier-Stokes solver. Recently, a new hybrid lattice Boltzmann numerical flux (HLBFS) is developed by Shu's group. It combines two different LBFS schemes by a switch function. It solves the Boltzmann equation instead of the Euler equation. In this article, the main object is to evaluate the ability of this HLBFS scheme by our in-house cell centered hybrid mesh based Navier-Stokes code. Its performance is examined by several widely-used bench-mark test cases. The comparisons on results between calculation and experiment are conducted. They show that the scheme can capture the shock wave as well as the resolving of boundary layer.
\end{abstract}

Keywords: Hybrid lattice Boltzmann numerical flux; aerodynamic forces; hybrid mesh.

\section{Introduction}

Computational fluid dynamics (CFD) has become an important tool in the engineering design. For example, the prediction of lift and drag plays an important role in the engineering design. There are many factors which affect the robustness of the calculation and the accuracy of the prediction. These factors include numerical convective schemes, methods for gradient calculation, the reconstruction methods and turbulence models etc. In this article, the attention is paid to numerical convective schemes. In fact, there are a lot of convective schemes developed so far. The upwind schemes can be grouped into flux vector splitting (FVS) and flux difference splitting (FDS). FVS (such as van Leer scheme $^{1}$ ) is famous for their robustness and efficiently capturing of strong shock wave. However, their intrinsic artificial dissipations are too big. This tends to artificially broaden boundary layers. Another upwind type is FDS such as Roe scheme ${ }^{2}$. It can be expressed as the average flux plus a dissipation term. It cannot properly identify an expansion fan that contains a sonic point and therefore an entropy correction is needed to

This is an Open Access article published by World Scientific Publishing Company. It is distributed under the terms of the Creative Commons Attribution 3.0 (CC-BY) License. Further distribution of this work is permitted, provided the original work is properly cited. 
break up expansion shocks. Besides, it suffers from the carbuncle problem. As compared to the traditional CFD schemes, the Boltzmann equation-based flux solver calculates the flux by solving the lattice Boltzmann equation. Recently, a new hybrid lattice Boltzmann numerical flux (HLBFS) ${ }^{3-4}$ is developed by Prof. Shu's group. It combines two different LBFS schemes by a switch function. It is not widely tested to show the aerodynamic performance especially the ability of resolving the boundary layer. Hence, in this article, it is to evaluate the ability of this HLBFS scheme by examining several widely-used bench-mark test cases.

\section{Methodology}

\subsection{Governing equations}

The compressible flows are solved by the Farve-averaged Navier-Stokes equations

$$
\partial_{t} \int_{\Omega} U d V+\int_{S} \Phi d S=0
$$

with turbulent modeling equations (S-A or SST etc.)

$$
\partial_{t} \int_{\Omega} M d V+\int_{S} \Psi d S=\int_{\Omega} S r c d V
$$

In Eq. (1), $U$ is the state vector, and $\Phi$ is flux vector,

$$
U=\left(\begin{array}{c}
\rho \\
\rho \vec{u} \\
E
\end{array}\right), \Phi=\Phi^{c}-\Phi^{v}, \Phi^{c}=u_{n}\left(\begin{array}{c}
\rho \\
\rho \vec{u} \\
E
\end{array}\right)+\left(\begin{array}{c}
0 \\
P \overrightarrow{\mathrm{n}} \\
P u_{n}
\end{array}\right), \Phi^{v}=\left(\begin{array}{c}
0 \\
{[\tau] \cdot \overrightarrow{\mathrm{n}}} \\
([\tau] \cdot \vec{u}) \cdot \overrightarrow{\mathrm{n}}+\vec{q} \cdot \overrightarrow{\mathrm{n}}
\end{array}\right)
$$

Here, $\rho$ is the density, $\vec{u}$ is the velocity, $\mathrm{E}$ is the total energy, $\mathrm{P}$ is the pressure, and $[\tau]$ is the stress tensor

$$
[\tau]=\left(\mu_{L}+\mu_{t u r}\right)\left[\nabla \vec{u}+\nabla^{T} \vec{u}-\frac{2}{3}(\nabla \cdot \vec{u})[\mathrm{I}]\right]
$$

\subsection{Numerical discretization}

Eqs. (1-2) can be discretized at cell $c$ by the multi-stage Runge-Kutta schemes

$$
U_{c}{ }^{(i)}=U_{c}{ }^{(0)}-\alpha_{i} \frac{\Delta t}{V^{n}} \operatorname{Res}_{c}{ }^{i-1}, i=1, \ldots, p-1
$$


with the residue defined as

$\operatorname{Res}_{c}=\sum_{f} \Phi_{f}\left(U^{-}, U^{+}, \vec{n}\right) \cdot A_{f}$.

Here, $V_{c}$ is the volume of cell, $\Phi_{f}\left(U^{-}, U^{+}, \vec{n}\right)$ is the numerical flux and $A_{f}$ denotes the area of the face $f$.

There are a lot of schemes implemented in the PolySim code. For the HLBFS scheme, it combines two different LBFS schemes

$\vec{\Phi}_{f}^{c}\left(U^{-}, U^{+}, \vec{n}\right)=(1-\alpha) \vec{\Phi}_{f}^{I}\left(f_{i}^{\text {interface }}\right)+\alpha \vec{\Phi}_{f}^{I I}\left(f_{i}^{\text {interface }}\right)$

by applying a switch function

$$
\alpha=\tanh \left(C \frac{\left|p_{l}-p_{r}\right|}{p_{l}+p_{r}}\right) \text {. }
$$

Here, the distribution function at the face is evaluated by

$$
f_{i}^{\text {interface }}=\left\{\begin{array}{ll}
f_{i}^{e q}(0, t) & \text { for scheme I } \\
f_{i}^{e q}\left(-\xi_{i} \delta, t-\delta\right) & \text { for scheme II }
\end{array} .\right.
$$

with the equilibrium function defined as

$$
\begin{aligned}
& f_{1}^{e q}=\rho \frac{-d_{2}^{2}\left(u_{n}+d_{1}\right)+d_{1}\left(u_{n}^{2}+c^{2}\right)+u_{n}\left(u_{n}^{2}+3 c^{2}\right)}{2 d_{1}\left(d_{1}^{2}-d_{2}^{2}\right)} \\
& f_{2}^{e q}=\rho \frac{d_{2}^{2}\left(u_{n}-d_{1}\right)+d_{1}\left(u_{n}^{2}+c^{2}\right)-u_{n}\left(u_{n}^{2}+3 c^{2}\right)}{2 d_{1}\left(d_{1}^{2}-d_{2}^{2}\right)} \\
& f_{3}^{e q}=\rho \frac{d_{1}^{2}\left(u_{n}+d_{2}\right)-d_{2}\left(u_{n}^{2}+c^{2}\right)-u_{n}\left(u_{n}^{2}+3 c^{2}\right)}{2 d_{2}\left(d_{1}^{2}-d_{2}^{2}\right)} \\
& f_{4}^{e q}=\rho \frac{-d_{1}^{2}\left(u_{n}-d_{2}\right)-d_{2}\left(u_{n}^{2}+c^{2}\right)+u_{n}\left(u_{n}^{2}+3 c^{2}\right)}{2 d_{2}\left(d_{1}^{2}-d_{2}^{2}\right)}
\end{aligned}
$$

Here, $d_{1}$ and $d_{2}$ are two lattice velocities used in the D1Q4 model. 


\section{Numerical Cases}

In order to evaluate the aerodynamic performance of this Hybrid lattice Boltzmann numerical flux, several test cases are examined.

\subsection{Turbulent boundary layer flows over a flat plate}

Turbulent boundary layer flow over a flat plate at a low Mach number is a common bench mark test. It has been extensively investigated by Wieghardt and Tillman ${ }^{5}$. The mesh used in this study is shown in Fig. 1. It consists of 5500 brick (hex) cells and 1860 prim cells. At the leading edge of the plate an additional part of mesh is used to buffer the incoming flow. Besides, clustered mesh points are generated before the leading edge (the beginning of the flat plate) to provide a uniform profile at the leading edge. Similarly, the grid was also clustered normally to the plate to resolve the boundary layer. The first cell height is small enough to capture the viscous sub-layer as well as the log layer. The upstream of the leading edge of the flat plate were treated as an in-viscid wall. The plate is a non-slip wall. The symmetric boundary condition is applied to the sidewalls.

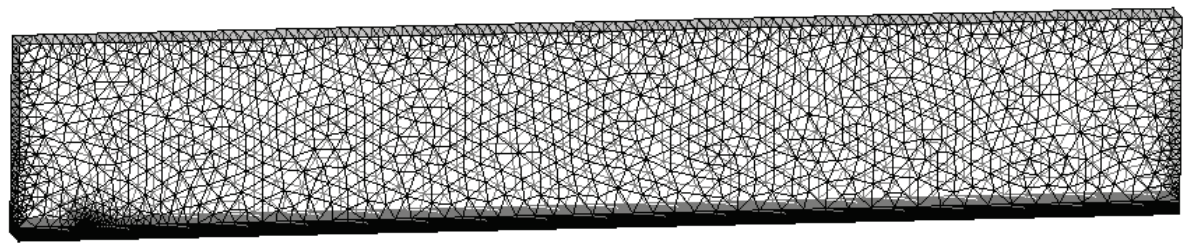

Fig. 1. Hybrid mesh for turbulent boundary layer flows over a flat plate.

The results are plotted in Fig. 2. It could be easily observed that the velocity profile agrees well with the wall laws. Besides, the skin friction profile is closed to the experimental one by Wieghardt and Tillman ${ }^{5}$.
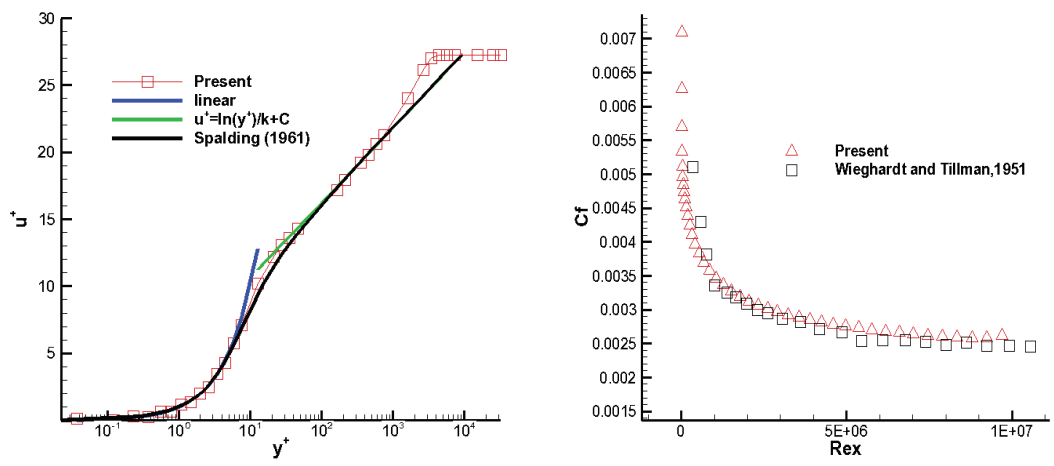

Fig. 2. The velocity and skin friction profiles. 


\subsection{Shock Wave Boundary Layer Interaction}

The study has been studied by Schulein et al. ${ }^{6}$ of German Aerospace Center (DLR). An oblique shock was created by a 10 degree oblique shock generator plate. This shock from tip of shock generator hits a long plate. After that, it interacts with the turbulent boundary layer developed over the flat plate. The length of this plate is $500 \mathrm{~mm}$. For this simulation, the free stream conditions are $\mathrm{M}_{\infty}=5, \mathrm{~T}_{\infty}=68.3 \mathrm{~K}$ and $\mathrm{p}_{\infty}=4008.5 \mathrm{~Pa}$. The hybrid mesh (Fig. 3) with hexahedral boundary layer cells are used in this study. The plate is an isothermal wall with $\mathrm{T}=300 \mathrm{~K}$. The symmetric boundary condition is applied to the sidewalls.

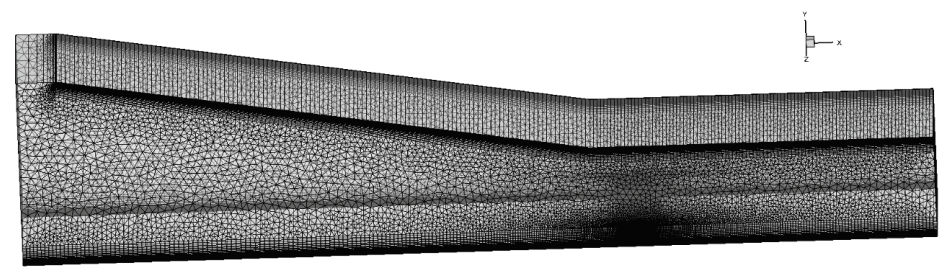

Fig. 3. Hybrid mesh for shock wave boundary layer interaction

The velocity profiles at different position are plotted in Fig. 4. It is clear that the velocity profile agrees well with the corresponding experimental one by Schulein et $\mathrm{al}^{6}$.
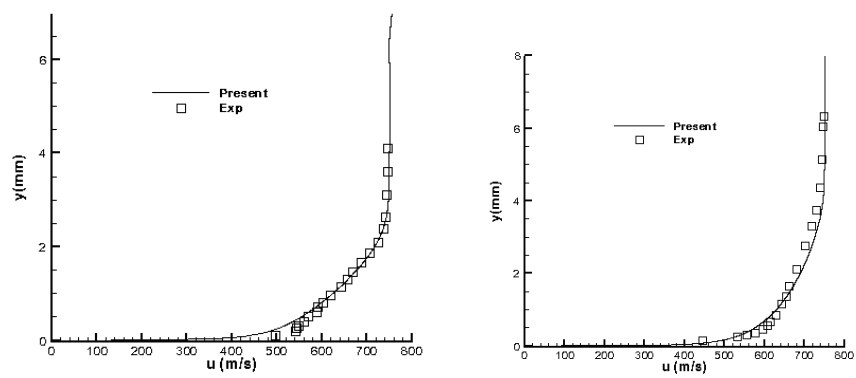

Fig. 4. Velocity profile at $\mathrm{x}=376 \mathrm{~mm}$ and $449 \mathrm{~mm}$.

\section{Conclusions}

The evaluation of the aerodynamic performance of hybrid lattice Boltzmann numerical flux is conducted. The two cases, turbulent boundary layer flows over a flat plate and the shock wave boundary layer interaction, are examined. The numerical results agree well with the experimental data. They show that the scheme can capture the shock wave as well as the resolving of boundary layer. 


\section{Acknowledgments}

This research work was supported by the Natural Science Foundation for the Youth of China under Grant 11202220.

\section{References}

1. B. van Leer, Flux vector splitting for the Euler equations, Lecture Notes in Physics, 170 507512 (1982).

2. P.L. Roe, Approximate Riemann solvers, parameter vectors, and difference schemes, J. Comput. Phys., 43, 357-372 (1981).

3. L.M. Yang, C. Shu, J. Wu, Development and comparative studies of three non-free parameter lattice Boltzmann models for simulation of compressible flows, Adv. Appl. Math. Mech., 4, 454-472 (2012).

4. L.M. Yang, C. Shu, J. Wu, A moment conservation-based non-free parameter compressible lattice Boltzmann model and its application for flux evaluation at cell interface, Comput. Fluids, 79, 190-199 (2013).

5. K. Wieghardt, and W. Tillman, On the Turbulent Friction Layer for Rising Pressure, NACA TM-1314 (1951).

6. E. Schulein, P. Krogmann, and E. Stanewsky, Documentation of Two-Dimensional Impinging Shock/Turbulent Boundary Layer Interaction Flow, DLR Report DLR IB 223-96 A 49, October (1996). 